\title{
Designs for Temperature and Temperature Gradient Com- pensated Capacitors Smaller Than Ten Picofarads
}

\author{
Robert D. Cutkosky \\ (July 22, 1964)
}

\begin{abstract}
A theoretical study is made of the dependence of capacitance upon electrode temperature in an air dielectric capacitor. The possibility of constructing a three-terminal standard whose capacitance depends only upon the temperature of one electrode is pointed out, and some practical capacitors utilizing the principle are described. The temperature sensitive electrodes of these capacitors are constructed of fused silica, which results in temperature coefficients of capacitance near $0.5 \mathrm{ppm} /{ }^{\circ} \mathrm{C}$. The temperature independent electrodes of these capacitors may be constructed of any stable metal, facilitating adjustment to nominal value.
\end{abstract}

\section{Introduction}

One of the problems encountered in the construction of standard air dielectric capacitors is that of reducing the dependence of capacitance upon temperature. The best technique seems to be to construct the capacitor entirely of materials which have very small mechanical temperature coefficients. Then provided that the electrode distortions and displacements caused by temperature dependences of the elastic coefficients of the electrodes and electrode supports are not significant, the capacitance temperature coefficient will also be small. Materials of small mechanical temperature coefficient which have been used for the construction of capacitors are fused silica and Invar. Fused silica is difficult to form and mount, and must be metalized to provide electrodes. Its exclusive use in a capacitor with air dielectric results in temperature coefficients of capacitance around $0.5 \mathrm{ppm} /{ }^{\circ} \mathrm{C}$. Invar is somewhat unstable and must be carefully heat treated. When treated for optimum stability, temperature coefficients around $+2 \mathrm{ppm} /{ }^{\circ} \mathrm{C}$ are typical.

The disadvantages of fused silica and Invar are often avoided by the use of two or more materials of different but relatively high temperature coefficients in an arrangement that provides temperature compensation. Temperature compensated capacitors using two metals have been built in many ways and with varying degrees of success. One simple but somewhat unsatisfactory design makes use of coaxial cylinders in which the outer cylinder has a higher temperature coefficient than the inner. The dimensions of the capacitor are chosen so that the capacitance between the cylinders is independent of temperature, provided that no temperature differential exists between the two cylinders.

Since the temperatures of the various elements in a capacitor are not generally equal but vary with the room or bath temperature with possibly different time lags, quite large cemperature differentials and capacitance changes can result. The cylindrical design is particularly objectionable from this standpoint, since the outer cylinder is often in more intimate contact with the surroundings than the inner cylinder, and hence responds to temperature changes more rapidly.

A temperature compensation technique often used for capacitors larger than $100 \mathrm{pF}$ involves stacking plates between spacers having a higher temperature coefficient than the plates. A second similar stack of plates is interleaved with, but insulated from, the first set of plates. Since the dissimilar metals are in intimate thermal contact, temperature differentials between plates and spacers remain relatively small, and the capacitance between the two stacks is not strongly affected by temperature fluctuations.

A more elaborate system of temperature compensation may be developed by considering the effect on the direct capacitance caused by independent variations in the temperatures of the separate electrodes. This procedure is found to be most useful for capacitors smaller than a few picofarads in which the temperature of the ground electrode may also be important.

\section{Partial Capacitance-Temperature Coefficients}

Consider a three terminal capacitor whose direct capacitance is given by $C=C\left(t_{1}, t_{2}, t_{3}\right)$ where the $t_{\text {'s }}$ are the temperatures of the three electrodes. Then for small departures from the reference temperature,

$$
C=C_{0}+\frac{\partial C}{\partial t_{1}} \Delta t_{1}+\frac{\partial C}{\partial t_{2}} \Delta t_{2}+\frac{\partial C}{\partial t_{3}} \Delta t_{3}
$$

or

$$
\begin{aligned}
C & =C_{0}\left(1+\frac{1}{C_{0}} \frac{\partial C}{\partial t_{1}} \Delta t_{1}+\frac{1}{C_{0}} \frac{\partial C}{\partial t_{2}} \Delta t_{2}+\frac{1}{C_{0}} \frac{\partial C}{\partial t_{3}} \Delta t_{3}\right) \\
& =C_{0}\left(1+\alpha_{1} \Delta t_{1}+\alpha_{2} \Delta t_{2}+\alpha_{3} \Delta t_{3}\right) .
\end{aligned}
$$


The terms $\frac{1}{C_{0}} \frac{\partial C}{\partial t_{1}}=\alpha_{1}$ etc., are the partial capacitancetemperature coefficients of the three electrodes.

If we let $l_{1}$ be a characteristic length of electrode 1 , we may write

$\alpha_{1}=\frac{l_{1}}{C_{0}}\left(\frac{\partial C}{\partial l_{1}}\right) \cdot \frac{1}{l_{1}}\left(\frac{\partial l_{1}}{\partial t_{1}}\right) \cdot$ We recognize $\frac{1}{l_{1}}\left(\frac{\partial l_{1}}{\partial t_{1}}\right)=\beta$ as

the thermal expansion coefficient of electrode 1 , and write

$$
\begin{aligned}
& \alpha_{1}=P_{1} \beta_{1} \text { where } P_{1}=\frac{l_{1}}{C_{0}} \frac{\partial C}{\partial l_{1}} \text {, etc., and obtain } \\
& \frac{C}{C_{0}}=1+P_{1} \beta_{1} \Delta t_{1}+P_{2} \beta_{2} \Delta t_{2}+P_{3} \beta_{3} \Delta t_{3} .
\end{aligned}
$$

If $\beta_{1}=\beta_{2}=\beta_{3}$ and $\Delta t_{1}=\Delta t_{2}=\Delta t_{3}$, we know from geometrical considerations that $\frac{C}{C_{0}}=1+\beta \Delta t$, and hence $P_{1}+P_{2}+P_{3}=1$, but no other restrictions on the $P$ 's exist.

Equation (2) suggests the possibility of designing capacitors with $P_{2}=P_{3}=0$ so that $P_{1}=1$. Then if $\beta_{1}$ is small, $C$ will be relatively independent of $\Delta t_{1}$, $\Delta t_{2}$, and $\Delta t_{3}$. This can be done by constructing electrode 1 of fused silica and choosing a suitable geometry. Such a capacitor may be adjusted by machining operations on electrodes 2 and 3 , which may be of any stable metal, and the principle objection to the use of fused silica is eliminated.

The above analysis is correct if no temperature gradients exist in any electrode, and if the capacitance changes caused by motions of the electrodes with respect to each other are not appreciable. The first criterion can be partially satisfied by avoiding the use of long, thin electrodes. The second criterion can be satisfied by locating the electrodes at saddle points in the capacitance-displacement function.

Experimentally it is often convenient to set one of the $P$ 's, say $P_{2}=0$, by a geometrical consideration, and then either to adjust $P_{3}=0$ or $P_{1}=1$ by observing the capacitance changes brought about by changing the dimensions of electrode 1 or electrode 3 . The easiest approach will be determined by the capacitor configuration.

\section{Guard-Cylinder Capacitor}

Consider a guarded cylindrical capacitor whose inner electrode has a radius $a_{1}$ and whose outer electrode and guards have a radius $a_{2}$. If the length of the outer electrode is $l$ and if $\epsilon$ is the permittivity of the dielectric, the direct capacitance of this system is given by

$$
C=\frac{2 \pi \epsilon l}{\ln \left(\frac{a_{2}}{a_{1}}\right)} .
$$

Differentiation yields

$$
\frac{C}{C_{0}}=1+\frac{1}{\ln \left(\frac{a_{2}}{a_{1}}\right)} \frac{\Delta a_{1}}{a_{1}}-\frac{1}{\ln \left(\frac{a_{2}}{a_{1}}\right)} \frac{\Delta a_{2}}{a_{2}}+\frac{\Delta l}{l} .
$$

For a uniform expansion of the outer cylinder, $\frac{\Delta a_{2}}{a_{2}}=\frac{\Delta l}{l}$, and $C$ is independent of the temperature of the outer cylinder if $\ln \frac{a_{2}}{a_{1}}=1$, or $\frac{a_{2}}{a_{1}}=e$. This relationship results in temperature compensation for the outer cylinder, but only if the guard expands at the same rate as the guarded electrode; and results in a capacitor whose temperature coefficient of capacitance is equal to the thermal expansion coefficient of the inner cylinder.

Although independent compensation for guard and guarded electrode temperature changes cannot be achieved with the simple guard cylinder capacitor, some insight into the behavior of more complicated cylindrical systems is derived from the results obtained. The critical ratio of radii, which is $e$ for the simple guard cylinder capacitor, is found to be quite close to this value for a large variety of completely compensated cylindrical designs.

\section{Practical Compensated Capacitors}

After some model studies, an experimental $5 \mathrm{pF}$ capacitor was constructed as shown in figure 1 . The inner electrode was a metallized fused silica cylinder. The guard electrodes were set back as illustrated to reduce the influence of their temperatures upon the direct capacitance of interest. It was found experimentally that small changes in the length of electrode 2 did not appreciably affect the compensation, so that adjustment to nominal value is simple.

Tests of the completed capacitor showed an overall temperature coefficient less than $1 \mathrm{ppm} /{ }^{\circ} \mathrm{C}$ and no appreciable overshoot when subjected to a rapid temperature change.

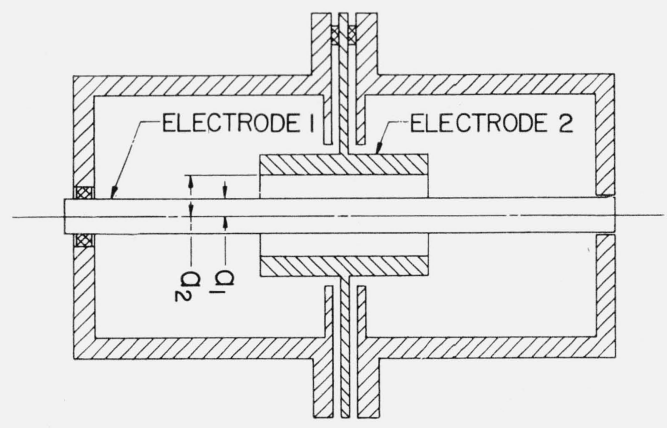

Figure 1. An experimental 5 pF completely temperature compensated capacitor drawn roughly to scale for correct compensation, $a_{2} / a_{1} \approx 2.5$. 
It is found that the design of figure 1 is not easily modified to yield either much larger or much smaller capacitance. Other designs have been investigated, however, which may be useful for the construction of arbitrarily small completely compensated capacitors. One such design is sketched in figure 2 . It makes use of a cylindrical shield between the two capacitor electrodes. The inner electrode is once again constructed of metalized fused silica. Holes in the cylindrical shield may be varied in sizes to provide a range of capacitances between the inner and outer electrodes. The outer electrode is automati-

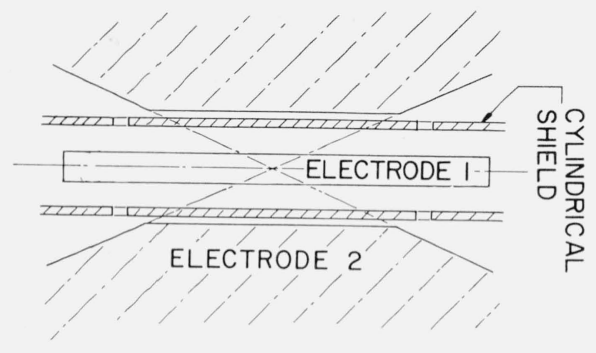

FIguRE 2. A completely temperature compensated capacitor for values smaller than $0.1 \mathrm{pF}$. cally temperature compensated by virtue of its shape. Any increase in its temperature will increase the spacing between it and the cylindrical shield, but will not change the position of the critical conical surfaces. The outer ends of the cones must of course be truncated, but it is found that this can be done without greatly upsetting the compensation. Experimentally it is found that the critical ratio of cylindrical shield radius to inner electrode radius is somewhat dependent upon the size and shape of the holes in the shield, but remains remarkably close to $e$.

\section{Conclusions}

Most practical capacitors are found to be not greatly dependent upon temperature differentials. However, complete disregard for the principles of partial temperature compensation can result in capacitors which are unduly sensitive to rapid temperature changes. It is possible at least in principle to design capacitors which depend only upon the temperature of one element; and if this element has a small thermal expansion coefficient, very close temperature control is not necessary. The technique seems most practical for capacitors smaller than $10 \mathrm{pF}$.

(Paper 68D4-177) 